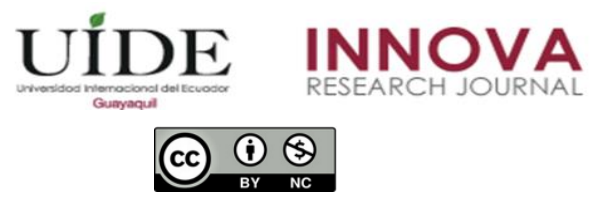

INNOVA Research Journal, ISSN 2477-9024

(Septiembre-Diciembre, 2019). Vol. 4, No.3 pp. 85-93

DOI: https://doi.org/10.33890/innova.v4.n3.2019.949

URL: http://revistas.uide.edu.ec/index.php/innova/index

Correo: innova@uide.edu.ec

\title{
El papel de las PYMES en las sociedades y su problemática empresarial
}

\section{Pymes' role withint the societies and its business issues}

Luis Eduardo Solis Granda

Rosa Claudiana Robalino Muñiz

Universidad Estatal de Milagro, Ecuador

Autor para correspondencia: 1solisg@unemi.edu.ec; rrobalinom@unemi.edu.ec

Fecha de recepción: 1 de febrero del 2019 - Fecha de aceptación: 1 de agosto del 2019

Resumen: Las pequeñas y medianas empresas (PYMES) representan un parte importante de la economía de un país, alrededor del $80 \%$ de las empresas constituidas en el Ecuador corresponden a PYMES y ésta conducta se observa en los demás países del mundo, pero lamentablemente se encuentran deficiencias en la gestión administrativa lo que ocasiona que ante la sociedad sean consideradas como empresas que no maduran o desarrollan económicamente. El objetivo de esta investigación es indagar sobre los procesos de gestión y la problemática de las PYMES a través de una investigación documental y deductivo-cuantitativo ya que en los últimos años la revisión de literatura, el debate y discusión sobre el tema en mención se ha ampliado y profundizado. Los resultados obtenidos de investigación revelaron: (1) retraso tecnológico (2) falta de asociatividad de las PYMES evidenciando, una deficiencia en la gestión interna y dificultad para enfrentar las competencias nacionales e internacionales. El aporte de esta investigación se centra en ahondar en las causas específicas de estos problemas, para determinarlas y puedan ayudar a mejorar el desarrollo productivo de los empresarios y microempresarios.

Palabras Claves: pymes; sociedad; problemas de negocio; falta de innovación

Abstract: Small and medium-sized enterprises (PYMES) represent an important part of a country's economy, around $80 \%$ of the companies constituted in Ecuador correspond to SMEs and this behavior is observed in other countries of the world, but unfortunately deficiencies are found in the administrative management what causes that before the society they are considered like companies that do not mature or develop economically. The objective of this research is to investigate the management processes and the problems of SMEs through a documentary research and deductive-quantitative analysis because in recent years the literature review, discussion and discussion on the subject in question has been extended and deepened. The results obtained from research revealed: (1) technological delay (2) lack of associativity of SMEs evidencing, a deficiency in internal management and difficulty in facing national and international competitions. The contribution of this research focuses on delving into the specific causes of these problems, to determine them and can help improve the productive development of entrepreneurs and microentrepreneurs.

Key Words: pymes; the societies; business issues; the lack of innovative 


\section{Introducción}

Según (Romero, 2006) la forma en valorar el papel desempeñado por las PYMES en el desarrollo económico ha estado sujeta a una evolución pendular a lo largo de las últimas décadas. En los años cincuenta y sesenta del siglo pasado, la pequeña empresa llegó a ser considerada una auténtica "distorsión del proceso de desarrollo" que se identificaba con la gran empresa y la concentración del capital. De igual manera (Tello, 2014) indica que la importancia de la PYME se evidencia desde los distintos ángulos. En el primer ángulo toma en cuenta a las PYMES como una de las principales fuentes de empleo; es interesante pues como herramienta de promoción de empleo en la medida en que solo exige una inversión inicial y permite el acceso a estratos de bajos recursos. En el segundo ángulo, puede potencialmente constituirse en apoyo importante a la gran empresa resolviendo algunos cuellos de botella en la producción. Los procesos administrativos, de gestión y los problemas que enfrentan las PYMES se refieren a las actividades que realizan dentro de una empresa a fin de lograr los objetivos planteados, pero que se ven obstaculizados por los problemas que enfrenta cada una de las organizaciones que conforman las PYMES.

Según (Torres, 2017) lo importante en el desarrollo de los procesos de gestión son los resultados, los mismos que deben ser lo más eficientes y deben generar seguridad en alcanzar los estándares de calidad.

En la actualidad gran parte de las empresas se ven desmotivas al generar nuevos productos para los clientes y esto se debe a que han sufrido pérdidas en productos que requerían de mucha inversión, pero no tuvo la acogida que se esperaba y esto se debe exclusivamente a que los productos no llenaron las expectativas de los consumidores (Filion, Cisneros, \& Mejia, 2011)

La pequeña y mediana empresa se ha constituido en objeto central de estudio de la teoría administrativa debido a que tienen una representación significativa en las economías de un país. Las investigaciones se han centrado fundamentalmente en el análisis de la perspectiva económica y en el ámbito de la gestión empresarial. (Guerrero, 2015)

Para el estudio de la problemática de las PYMES mezclan dos enfoques definidos como externos e internos; el enfoque externo identifica los problemas donde las Pymes no tienen control es decir en el sentido macroeconómico, en cambio el interno determina los problemas de gestión que sufre la empresa, los mismos que podrían tener una solución.

A través de los enfoques antes mencionados se puede llegar a identificar cuáles han sido los factores que generan problemas que afectan a los negocios a nivel interno, (Palomo, 2005) "resalta la falta de capacitación y la falta de una cultura de innovación y desarrollo tecnológico como los principales problemas de las empresas estos problemas varían según el tamaño y la actividad que ejecute".

(Guerrero, 2015) En su investigación menciona cuatro aspectos de la problemática; El paradigma manejado por los dueños, asumiendo la gerencia siendo la falta de tiempo una excusa para planear ni hacer inversiones e investigaciones para el desarrollo de la misma, la influencia 
del entorno, las deficiencias en la gestión interna y la incapacidad para asociarse y formar clusters que les permitan aunar esfuerzos para enfrentar la competencia nacional y transnacional.

De tal manera se puede asumir a través de estos dos estudios que llegan a la conclusión de que la deficiencia de gestión interna es uno de los factores más importantes pues le generan problemas a las pymes, pero al ser éste un factor interno puede identificarse como: la falta de capacitación y la falta de una cultura de innovación y desarrollo tecnológico, la forma de resolverse es tomando medidas correctivas para que la empresa alcances sus objetivos organizacionales y sobre todo siga en crecimiento, lo cual no solo se beneficia a si misma sino también al país de manera que éstas generan empleo y forman parte considerable del PIB del estado.

Posteriormente estas empresas pueden adoptar estrategias que les proporcione un aporte a la solución tomada para resolver los problemas que tiene la empresa, según (Parada \& Madriz, 2012) una de las estrategias más importantes para las PYMES es la planificación operativa, pues sólo se establecen objetivos y metas a corto plazo, a fin de minimizar la incertidumbre y generar fortalezas para enfrentar los retos que se les presenta con base en los intereses de diversificación en el mercado.

Esta investigación tiene por objeto indagar sobre los procesos de gestión y la problemática de las PYMES en el Ecuador, partiendo de esta sección introductoria y considerando que las organizaciones no sólo deben convivir y deleitarse con los éxitos del presente, sino seguir soñando y planificando un futuro prometedor.

\section{Definiciones de PYMES}

Para entender mejor lo que son las PYMES citaremos a continuación varias definiciones; PYMES quiere decir Pequeñas Y Medianas Empresas. En Ecuador, se llama PYMES al conjunto de pequeñas y medianas empresas, que, de acuerdo al número de trabajadores, volumen de ventas, años en el mercado, y sus niveles de producción, activos, pasivos (que representan su capital) tienen características similares en sus procesos de crecimiento. (Enroke, 2015)

En la clasificación de las empresas de acuerdo a su tamaño, se consideran pequeñas a las compañías con ingresos que oscilan USD 100.000 (Cien Dólares) y USD 1.000.000 (Mil dólares) anuales. Mientras que las medianas son aquellas que registran valores entre USD 1.000.000 (Mil dólares) y 10.000.000 (Diez mil dólares). (Ekos, 2017)

Según las investigaciones sobre las Pymes, desarrolladas por el Instituto Nacional de Estadísticas y Censos (INEC), unas de las características fundamentales de éste tipo de empresas es el uso intensivo de la mano de obra, en segundo lugar se encuentran la poca aplicación de la tecnología, y de ahí en importancia se encuentran también: la baja división del trabajo, reducido capital, baja productividad, mínima capacidad de ahorro y limitado uso de los servicios financieros y no financieros. . (INEC, 2012)

Las pequeñas y medianas empresas se han catalogado en el mundo en desarrollo como organizaciones muy importantes para la economía en general y para la sociedad en particular por 
su potencial de generar empleo, por su capacidad de producir ingreso para los sectores débiles, por ampliar la base del sector privado, por contribuir a reducir la concentración de poder económico y por su aporte al producto nacional. (Castellanos, 2003)

Para entender mejor lo que son las PYMES podemos también basarnos en el siguiente concepto entablado por (Vera Colina, Melgarejo Molina, \& Mora Riapira, 2014) se trata de un sector caracterizado por un alto dinamismo y flexibilidad en sus operaciones, pero al mismo tiempo marcado por un conjunto de dificultades que tienden a obstaculizar su sostenibilidad y crecimiento.

\section{Importancia de la PYMES}

Una vez conocidas diferentes definiciones de la PYMES concluimos que estas son de suma importancia para el desarrollo de un país, ya que promueven a la reducción de la tasa de desempleo mediante los también conocidos como "micro emprendedores" donde aportan de manera considerable al Producto Interno Bruto (PIB).

Según (Enrique, 2012) Las Pymes deben ser consideradas organizaciones pequeñas, con rasgos sociales, que tiene como objetivo económico sostenerse en el mercado, con el fin social de mantener y dar prosperidad a la familia.

Por otro lado, (Romero, 2006) La valoración del papel desempeñado por las PYME en el desarrollo económico ha estado sujeta a una evolución pendular a lo largo de las últimas décadas. En los años cincuenta y sesenta del siglo pasado, la pequeña empresa llegó a ser considerada una auténtica "distorsión del proceso de desarrollo" que se identificaba con la gran empresa y la concentración del capital debido a que muchas de éstas organizaciones pequeñas se constituyeron familiarmente.

De igual manera (Tello, 2014) indica que la importancia de la PYME se evidencia desde los distintos ángulos. En el primer ángulo toma en cuenta a las PYMES como una de las principales fuentes de empleo; es interesante pues como herramienta de promoción de empleo en la medida en que solo exige una inversión inicial y permite el acceso a estratos de bajos recursos. En el segundo ángulo, puede potencialmente constituirse en apoyo importante a la gran empresa resolviendo algunos cuellos de botella en la producción

- Para (Nuño, 2012) El gran reto de la pequeña empresa es mantenerse viva, en un ambiente que la reta a luchar día con día, con el apoyo de estrategias, capital humano, métodos, técnicas y procesos; los principales retos a los que se enfrenta son:

$\checkmark$ La nueva economía de globalización provoca un alto nivel de competencia

$\checkmark$ El aumento en la complejidad de los mercados.

$\checkmark$ Excesivos controles gubernamentales y altas tasas impositivas.

Formar profesionales capaces de romper con los paradigmas organizacionales que permitan transformar las empresas emergentes y confiables en competentes. 
En cambio, , (Navarro, García, \& Vela, 2009) La fuerte presencia de las PYMES en la Comunidad Europea, representan más del $90 \%$ de las firmas de la Comunidad y aseguran más de dos tercios del empleo europeo, siendo todavía mayores estas cifras en nuestro país.

(Fedmantar, 2014) Según la Secretaría de Economía, a través de la Subsecretaría para la Pequeña y Mediana Empresa (SPyME), por ejemplo se observa en México que existen aproximadamente 4 millones 15 mil empresas, donde el 99.8\% son Pymes. Se puede decir que el país no tiene políticas adecuadas, y las empresas solo tratan de mantenerse reduciendo el costo de sus ventas para generar mayores ingresos.

\section{Problemática de la PYMES}

Dentro del contexto en el que se desarrollan las organizaciones en su totalidad, se encuentran las pequeñas y medianas empresas, éstas son importantes porque permiten incrementar el desempeño económico del país, a través de la generación de recursos monetarios y el aumento de empleo (Velásquez, 2003) sin embargo, estas empresas presentan ciertas problemáticas en relación a su operatividad, las cuales afectan al desarrollo de dichas empresas y a su vez al tejido económico del país en que se encuentran.

Según (Palomo, 2005) las pequeñas y medianas empresas presentan problemas de carácter interno y externo, estas problemáticas merman el crecimiento empresarial, por lo cual, el estudio y análisis de dichas variables ayudan a reducir las incertidumbres que se encuentren en los procedimientos que opera la entidad, por otro lado, enfatiza en que se debe priorizar el estudio de los problemas internos (gestión interna), ya que, en el contexto externo, las pymes no pueden ejercer ningún efecto en su reducción.

Este tipo de empresas se enfrentan a varios factores que influyen significativamente en el desarrollo de las mismas los conocidos factores externos son los que se relacionan con las PYMES, como el entorno económico, los impuestos, subvenciones se caracterizan porque los cambios que estas empresas realicen no afecta en nada a dichos factores, por lo contrario los factores internos están estrechamente relacionados con la empresa ya sea por problemas de gestión propia del negocio, la organización, el modelo de negocio, estos factores se caracterizan porque la empresa es responsable de la solución y mejora. ( Barajas, Hunt, \& Riba, 2013)

Los factores de la gestión interna de las empresas según (Heizer \& Render, 2009) se resumen en tres vertientes: 1. Producción y operaciones (Fabricar, comprar, vender, trasportar, etc.) 2. Finanzas y contabilidad (registros contables, estados financieros, etc.) y 3. Marketing (creación de valor en los productos a comercializar). A partir de esta descripción (Palomo, 2005) indica que "los procesos de gestión pueden dividirse en dos tipos: procesos operativos y los procesos de apoyo" (p. 30).

(Jacques, Cisneros, \& Mejía, 2011) indica que algunos administradores consideran que las operaciones son procedimientos a los que no tenemos que prestar atención ya que frena el impulso de los emprendedores, sin embargo, las operaciones pueden incrementar la ventaja de competitivad que posee una empresa ya que la adecuada organización de los recursos, elementos y procesos dentro de las entidades, tanto industriales, de servicio, o comerciales, ayudan a 
optimizar la generación de recursos monetarios, lo cual permite que las empresas pequeñas, crezcan, se adapten y creen redes de producción y distribución eficaces y eficientes.

Así mismo, (Chase, Jacob, \& Aquilano, 2009) manifiesta que existe una relación entre los negocios y los enfoques para administrar procesos de operaciones, ya que dichas operaciones son creadas a partir de una red sistematizada de procedimientos, en las cuales las pequeñas y medianas empresas presentan irregularidades en su gestión, por lo que, la mejora de dichos procesos se puede transformar en reducción de costos de operación y maximización de beneficios económicos. Metodología

En tanto que el estudio trata de aportar conocimiento sobre la importancia de las PYMES y su problemática empresarial, que ayuden a mejorar el desarrollo productivo de los empresarios y microempresarios, metodológicamente la investigación aplicada fue la documental que se basa en el análisis de texto, artículos científicos y material bibliográfico que fueron utilizados para profundizar los temas de la investigación.

La investigación tiene dos métodos afines a sus características particulares, el primero a través del análisis documental con el cual se pudo recabar información para el posterior análisis de la importancia de las PYMES y su problemática empresarial, el segundo método es un método deductivo - cuantitativo que considera que la conclusión se halla implícita dentro las premisas. (Definción.de, 2012) Reflexiones finales

Por tanto, en base a la información revisada de los autores citados en párrafos anteriores se puede concluir que existe una gran deficiencia en la gestión administrativa de las PYMES y los problemas se debe a la falta de organización administrativa, coordinación en las actividades, incentivos a los trabajadores, falta de políticas escritas, falta de supervisión, control en consecuencia debido a retraso tecnológico que éstas tienen. Provocando una inexistencia del crecimiento de las empresas es consecuencia de un recurso humano conformista y desmotivado, un recurso humano que es pobre de conocimiento debido a que el empleador no los capacita.

También se puede deducir que las PYMES comenten muchos errores al promocionar un producto o un servicio que en muchas ocasiones no satisface ninguna necesidad o existen mejores productos que el que ellos ofertan o en ciertos casos enfaticen el producto y no en la satisfacción de las necesidades del consumidor que es lo que realmente importa.

Las Pymes son la fuente de empleo para millones de personas por esta razón es muy importante que los propietarios hagan conciencia de la situación actual de las empresas y tomen las medidas necesarias para evitar que estas desparezcan, ya que si esto llegase a pasar sería un desastre fatal porque millones de personas quedarían desempleadas y esto representa un gran daño para la sociedad en general.

Las Pymes son empresas que contribuyen al desarrollo económico del país, por lo que es primordial que realicen sus operaciones de forma eficiente, logrando una estabilidad entre la organización y sus factores externos e internos, por lo cual, se debe analizar en mayor proporción el impacto que tienen los factores internos (controlados y manejados por la organización) en la generación de recursos monetarios. 
Las PYMES constituyen una parte fundamental en los países, principalmente latinoamericanos, ya que generan plazas de empleo y mueven la economía interna del país y del sector en donde estos se desarrollan.

El sector de las PYMES ha tomado fuerzas en la última década, a nivel nacional, convirtiéndose en la actividad principal de cada ciudad, constituyéndose como una fuente relevante de dinero, incentivando al movimiento del efectivo local y nacionalmente.

Las Pymes presentan una alta participación en el mercado y en la economía nacional, por lo que se constituye en un actor fundamental en la generación de riqueza y empleo por esto, lo cambiante del entorno económico en que operan las Pymes, hace que éstas se vuelvan versátiles y se adapte su gestión, estructuras y procesos, aunque existen diversos obstáculos que impiden a que sigan adelante los cuales pueden sobrellevarlos mediante una buena estructura organizacional.

En conclusión es necesario buscar alternativas de solución que permita la supervivencia de las empresas PYMES dentro del mercado y una de las alternativas más viables para el logro de objetivos, es realizar procedimientos de gestión interna como sondeos y nichos de mercado para conocer las necesidades y expectativas de sus posibles clientes atendiendo mejor a las demandas. Sus clientes pueden recibir un trato más personalizado. En el análisis se observa que sus mayores obstáculos son el retraso tecnológico y la falta de innovación lo que ocasiona a las PYMES menores posibilidades de expansión y la falta de asociatividad de las mismas.

Cabe mencionar (Kawasaki, 2007) En su obra el Arte de empezar menciona "PIENSA A LO GRANDE. Ponte el listón bien alto y lucha por algo grande. Si vas a cambiar el mundo, no puedes hacerlo con productos y servicios aburridos o insignificantes. Ve por cosas que sean, por lo menos, diez veces mejores de lo que ya existe. En este contexto deja muy claro que para que un producto o servicio tenga éxito debe ser de calidad y resaltar ante los demás, es aquí donde interviene la gestión administrativa, son ellos quienes deben motivar al talento humano a generar y brindar servicio de calidad y abastecer de todo lo necesario para que los trabajadores se sientan a gusto con su trabajo. (Valencia, 2010) manifiesta que la columna vertebral de una empresa son los empleados por esta razón fundamental que ellos trabajen en condiciones óptimas, capaciten al personal, adquieran el financiamiento necesario para alcanzar las metas planteadas y no ser uno más de los emprendedores que empezaron un negocio y luego lo abandonaron para que una empresa tenga éxito debe priorizar las necesidades y los clientes de esa manera el producto que desarrolles de seguro les encantara. (Sánchez, Salazar, \& Soto, 2014)

Podemos decir que las PYMES en la mayoría de los países son muy características en la economía, ya que estas son las micro, pequeñas y medianas empresas, enfocándonos en el caso del Ecuador estas han enfrentado un escenario complicado tanto por la situación económica como por la falta de políticas de incentivos a la producción que se registraron. El dinamismo de una economía depende de la capacidad de promover, sustentar y reconocer el crecimiento de los negocios, algo que aún debe trabajarse con gran énfasis en cada una de las regiones del Ecuador, ya que esto permitirá la mejora económica de nuestra Nación. 


\section{Bibliografía}

Barajas, S., Hunt, P., \& Riba, G. (2013). Las finanzas como instrumento de gestión de las pymes: Un manual divulgativo para los propietarios, gerentes y gestores de pymes.

Castellanos, J. (2003). Pymes Innovadoras. Cambios de Estrategias e Instrumentos. Escuela de Administración de Negocios.

Chase, R., Jacob, F., \& Aquilano, N. (2009). Administración de operaciones: producción y cadena de suministros. México: McGraw-Hill.

Definción.de. (2012). Definición. Obtenido de https://definicion.de/metodo-deductivo/

Ekos. (31 de octubre de 2017). Ekos Negocios. Obtenido de http://www.ekosnegocios.com/negocios/verArticuloContenido.aspx ?idArt=9813

Enrique, L. C. (2012). Influencia del capital humano para la competitividad de las pymes en el sector manufacturero de Celaya, Guanajuato. Obtenido de http://www.eumed.net/tesisdoctorales/2013/jelc/index.htm

Enroke. (2015). Grupo Enroke. Obtenido de http://www.grupoenroke.com/index.php/proyectopymes/46-que-son-las-\%20pymes

Fedmantar. (09 de enero de 2014). Club Ensayos. Obtenido de https://www.clubensayos.com/Negocios/Ensayo-Sobre-Los-Problemas-Que-EnfrentanLas-PyMES/1372681.html

Filion, L., Cisneros, L., \& Mejía. (2011). Administración de Pymes. (Pearson, Ed.) Obtenido de http://cpx.mx/acabrera/bStarter/Administracion_de_PYMES.pdf

González, M. (2005). Los procesos de gestión y la problemática de las Pymes. Obtenido de http://virtual.unemi.edu.ec/pluginfile.php/306669/mod_resource/content/0/LOS

Guerrero, Z. (2015). Las Pymes y su problemática empresarial. E.

Heizer, J., \& Render, B. (2009). Principios de Administración de Operaciones. México: Pearson.

INEC. (30 de julio de 2012). INSTITUTO NACIONAL DE ESTADISTICAS Y CENSOS. Obtenido de http://www.ecuadorencifras.gob.ec/institucional/home/

Jacques, L., Cisneros, L., \& Mejía, J. (2011). Administración de Pymes: Aprender, dirigir y desarrollar empresas. México: Pearson.

Julio. (Septiembre 2005). Proceso de gestión y problemática de las pymes (Vol. VIII).

Kawasaki, G. (2007). El arte de empezar. España: Pearson.

Keuffman. (2001). el impacto de las políticas de apoyo a la PYMES. Ajoica contenidos.

Navarro, E., García, M., \& Vela, M. (2009). ESIC MARKET. Obtenido de http://www.esic.edu/documentos/revistas/esicmk/060130_908283_E.pdf

Nuño, P. (2012). Administración de pequeñas empresas. Obtenido de DOI: https://doi.org/http://dx.doi.org/10.1016/0025-326X(91)90138-I

Palomo, M. (2005). Los procesos de gestión y la problemática de las Pymes.

Parada, \& Madriz. (2012). Estrategias para la adecuación teórica del proceso planificador en las microempresas manufacturas del estado Táchira.

Romero, I. (2006). Las Pyme en la economía global. Hacia una estrategia de Fomento Empresarial. Revista Latinoamericana de Economía, 31-50.

Sánchez, Salazar, \& Soto. (2014). El emprendimiento y crecimiento de las pymes. Obtenido de https://www.redalyc.org/articulo.oa?id=41648308006

Tello, S. (2014). Importancia de la micro, pequeñas y medianas empresas en el desarrollo del país. Obtenido de https://doi.org/10.21503/lex.v12i14.623 
Torres, J. (2017). Financiamiento Org. Obtenido de https://financiamiento.org.mx/pymes-enmexico/

Valencia, J. (2010). Administración de pequeñas y medianas empresas. México: Cengage Learning.

Velásquez, A. (2003). Modelo de gestión de operaciones para pymes innovadoras. Revista Escueal de Administración de Negocios, 47.

Vera Colina, M., Melgarejo Molina, Z., \& Mora Riapira, E. (2014). Acceso a la financiación en Pymes colombianas: una mirada desde sus indicadores financieros. INNOVAR. Revista de Ciencias Administrativas y Sociales, 149-160. 